\title{
Combining observations with acoustic swath bathymetry and backscat- ter to map seabed sediment texture classes: the empirical best linear unbiased predictor
}

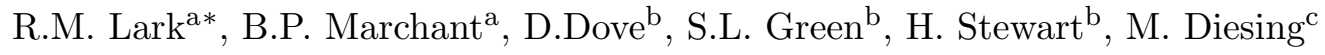 \\ ${ }^{\mathrm{a}}$ British Geological Survey, Keyworth, Nottingham, NG12 5GG, british Geological \\ Survey, Murchison House, Edinburgh, EH9 3LA, ${ }^{\mathrm{c}}$ Centre for Environment, Fisheries \\ and Aquaculture Science, Pakefield Road, Lowestoft, Suffolk NR3 30HT
}

\section{Abstract}

3 Seabed sediment texture can be mapped by geostatistical prediction from limited

4 direct observations such as grab-samples. A geostatistical model can provide local

5 estimates of the probability of each texture class so the most probable sediment

6 class can be identified at any unsampled location, and the uncertainty of this pre-

7 diction can be quantified. In this paper we show, in a case study off the northeast

8 coast of England, how swath bathymetry and backscatter can be incorporated into

9 a geostatistical linear mixed model (LMM) as fixed effects (covariates).

Parameters of the LMM were estimated by maximum likelihood which allowed us to show that both covariates provided useful information. In a cross-validation, each observation was predicted from the rest using the LMMs with (i) no covariates, or (ii) bathymetry and backscatter as covariates. The proportion of cases in which the most probable class according to the prediction corresponded to the observed class was increased (from $58 \%$ to $65 \%$ of cases) by including the covariates which also

increased the information content of the predictions, measured by the entropy of the class probabilities. A qualitative assessment of the geostatistical results shows that the model correctly predicts, for example, the occurrence of coarser sediment over discrete glacial sediment landforms, and muddier sediment in relatively quiescent,

*Corresponding author: E-mail address: mlark@bgs.ac.uk (R.M. Lark). 
localized deep water environments. This demonstrates the potential for assimilating geophysical data with direct observations by the LMM, and could offer a basis for a routine mapping procedure which incorporates these and other ancillary information such as manually-interpreted geological and geomorphological maps.

\section{Introduction}

Mapping benthic habitats is crucial for underpinning decision-making concerning management of the seabed. To encourage good practice and to ensure comparability across environmental and political boundaries, many practitioners use the hierarchical European Nature Information System (EUNIS) habitat classification scheme (e.g., Connor et al., 2006). The foundation of the EUNIS classification for the seabed is the seabed substratum type, because of the influence this has on the occurrence and abundance of benthic flora and fauna. Seabed habitats are principally split into rock and other hard substrata on the one hand, and sediment habitats on the other. Rock habitats have been identified as important for conservation due to their high biodiversity (e.g., Evans et al., 2015). However, they only account for a very limited area of seabed, which is typically dominated by sediment over large parts of the continental shelves. Mapping the distribution of seabed sediment classes can therefore serve as a proxy for regional benthic habitats (e.g., Kostylev et al., 2001; Howell, 2010; McGonigle and Collier, 2014). The distribution of seabed sediments is also of immediate interest to any industry (e.g., offshore wind, oil and gas) which seeks to install seabed infrastructure, where specific considerations may include sediment mobility, constraints on pile design, or pollutant dispersal (International Association of Oil \& Gas Producers, 2013).

The acquisition of seabed sediment samples is most commonly achieved by grab sampling, and samples are characterized through particle size analysis (PSA) using the 'Wentworth' textural classification (Wentworth, 1922). When mapping seabed 
sediment distribution, geologists commonly employ the Folk classification scheme (Folk, 1954) as it provides a useful indication of the energy of the seabed environment, but within the EUNIS habitat mapping classification the Folk scheme is simplified into only four textural classes which can be identified from the proportions of gravel, sand and mud size fractions among the mineral particles of the sediment (Long, 2006). The definitions of these classes are shown in Fig. 1 on the ternary diagram for gravel, sand and mud.

Lark et al. (2012) explored the use of geostatistical methods to predict the distribution of gravel, sand and mud fractions directly from point observations by compositional cokriging. However, this exploits only the spatial dependence of the variation of particle size fractions as shown by the data, and does not incorporate other types of relevant information. One example is data from swath bathymetry surveys. Both the bathymetry, and the associated intensity of the backscatter signal from the seabed, are informative about sediment texture (e.g., Goff et al., 2004; Fonseca et al., 2009).

Commonly, bathymetry and backscatter data are used to map the distribution of textural classes by expert interpretation. This interpretation is conducted in the light of the evolution of the seabed. When extrapolating the sample point data according to the geographically continuous acoustic data, the expert interpreter may consider, for example, how the interplay of the pre-existing geological substrate is modified over multiple climatic and environmental timescales (e.g., glaciation); and how the evolving seabed geomorphology is further impacted upon by active sedimentary, hydrodynamic, or even biological processes. There are many examples of such interpretative studies (e.g., Hughes Clarke et al., 1996; Kostylev et al., 2001; Glynn et al., 2015). Expert interpretation, however, is demanding of time. There is also an element of subjectivity in the results as they depend on judgements which may differ between individuals. Because of this, there has been considerable interest in developing quantitative techniques to establish predictive relationships between 
acoustic data and sediment properties (see review by Brown et al., 2011).

There are two general quantitative approaches. The first uses physical models of the backscattering of an acoustic signal by material on the seabed. At any location the sediment properties are found that give the best match between the predicted and observed backscatter (e.g., Sternlicht and de Moustier, 2003; Snellen et al., 2011). A second approach uses statistical predictive relationships. These may be semi-empirical, a predictive function with a physical interpretation is fitted statistically to observed data on sediment texture and the acoustic signal (e.g., Endler et al, 2015). Other statistical methods that have been applied include multivariate clustering (e.g., Anderston et al, 2002), clustering on multifractal properties of the time-dependent backscatter (Haris and Chakraborty, 2014), decision trees (e.g., Dartnell and Gardner, 2004), artificial neural networks (e.g., Marsh and Brown, 2009) and random forests (e.g., Lucier et al., 2013).

In a comparative study by Diesing et al. (2014) a range of mapping approaches was applied for interpretation of seabed sediments based on acoustic data (swath bathymetry, backscatter and derivatives of bathymetry such as rugosity which is a measure of high-frequency roughness) along with PSA samples. The approaches undertaken included expert interpretation, geostatistics (cokriging), object-based image analysis (OBIA) and random forests. In this case, the geostatistical method did not incorporate the acoustic data, whereas they were used by the machine learning, OBIA and expert interpretation techniques. However, these do not account explicitly for the spatial distribution of the observations, nor of the spatial dependencies among the data (although the more desirable properties of machine-learning methods such as random forests depend on the assumption that the observations are independent). Diesing et al. (2014) compared their results for prediction using acoustic data with the direct application of cokriging, as described by Lark et al. (2012). They found that a pixel by pixel comparison between the outputs resulted in agreements of between $68.1 \%$ and $73.1 \%$ for the cokriging method when compared 
with the mapped outputs from the other techniques. There was a marked difference between this method and those which utilised the acoustic data, with the major differences resulting from the less frequently occurring sediment classes.

In this paper we consider the possibility of combining linear models for the relationship between particle size distribution and variables derived from acoustic data with the cokriging procedure to account for the remaining unexplained variation. This is done by fitting a linear mixed model (LMM) for the (appropriately transformed) particle size distribution of sediment with fixed effects (covariates) the bathymetry and backscatter from the acoustic survey, and a suitable linear model of coregionalization for the residuals. The prediction conditional on this model is the empirical best linear unbiased predictor with the mean vector of the dependent variables a function of the covariates. We compare this result with the ordinary cokriging method in which the mean vector is assumed to be constant.

\section{Methods}

\subsection{The statistical model}

\subsubsection{Compositional data and their treatment}

Seabed sediment texture classes are defined according to the proportions by mass of gravel, sand and mud in the sediment on the logarithmic Wentworth scale (Wentworth, 1922). The size classes are exhaustive, and so the proportions of gravel, sand and mud necessarily sum to one. This makes the variate a composition (Aitchison, 1986) which is constrained to a distribution on the two-dimensional simplex, and which can be plotted as a ternary diagram.

The compositional nature of data such as those on sediment particle sizes must be accounted for in analysis. The problem is that, because the gravel, sand and mud proportions sum to one, they cannot vary jointly like other multivariate data sets, and spurious correlations can emerge between variables. The methods to deal with this problem that Aitchison (1986) proposed are based on taking ratios between compositional variables. Whereas sand and mud proportions cannot vary indepen- 
dently (if, for example, we know that the mud proportion is 0.7 then we know that the sand proportion is less than or equal to 0.3 ), their respective ratios to the gravel proportion are not so constrained. These ratios are generally transformed to logarithms to make an assumption of a joint normal distribution plausible. These are called the additive log-ratios of the compositional variables.

Lark et al. (2012) used compositional cokriging, as described by PawlowskyGlahn and Olea (2004), to predict seabed sediment texture by ordinary cokriging of the additive log-ratios. The additive log-ratio (ALR) transform can be applied to an $m$-part composition to create $m-1$ new variables which are not constrained onto a $m$-1-dimensional simplex but can be regarded as an unconstrained $m-1$ dimensional variate.

In the case of a three-variate compositional random variable $\mathbf{Z}=\left\{Z_{1}, Z_{2}, Z_{3}\right\}$ a corresponding two-dimensional ALR variate is $\mathbf{Y}=\left[Y_{1}, Y_{2}\right]^{\mathrm{T}}$, where

$$
\begin{aligned}
Y_{1} & =\ln \frac{Z_{1}}{Z_{3}}, \\
Y_{2} & =\ln \frac{Z_{2}}{Z_{3}} .
\end{aligned}
$$

Note that the selection of a variable from the compositional variate to serve as the denominator of the log-ratio does not affect the final outcome of analyses (PawlowskyGlahn and Olea, 2004). The ALR transform can be inverted:

$\mathbf{Z}=\left\{\frac{\exp \left(Y_{1}\right)}{1+\exp \left(Y_{1}\right)+\exp \left(Y_{2}\right)}, \frac{\exp \left(Y_{2}\right)}{1+\exp \left(Y_{1}\right)+\exp \left(Y_{2}\right)}, \frac{1}{1+\exp \left(Y_{1}\right)+\exp \left(Y_{2}\right)}\right\}^{\mathrm{T}}$,

, where the superscript $\mathrm{T}$ indicates the transpose of the matrix.

\subsubsection{The linear mixed model for compositional data}

Lark et al. (2012) used cokriging of the ALR-transform of the particle size composition. They sampled the prediction distribution of the ALR-transformed variate at the nodes of a prediction grid to estimate the probability at each node that each of the EUNIS sediment texture classes occurred. In this paper we extend this methodology to include bathymetry and backscatter as predictive covariates. 
This is done in the linear mixed modelling framework following the methods of Marchant and Lark (2007).

Consider a simple case where a two-dimensional ALR variate, $\mathbf{Y}$, is to be modelled as a linear function of some covariate $X$. We have $n$ collocated observations of the two ALR variables which we denote by the $n \times 1$ vectors $\mathbf{y}_{1}$ and $\mathbf{y}_{2}$, let $\mathbf{x}$ denote the $n \times 1$ vector of corresponding observations of the covariate, and let $\mathbf{1}_{n}$ denote a $n \times 1$ vector of ones. The LMM for $\mathbf{Y}$ is

$$
\left[\begin{array}{l}
\mathbf{y}_{1}^{\mathrm{T}} \\
\mathbf{y}_{2}^{\mathrm{T}}
\end{array}\right]=\left[\begin{array}{ll}
\alpha_{1} & \beta_{1} \\
\alpha_{2} & \beta_{2}
\end{array}\right]\left[\begin{array}{l}
\mathbf{1}_{n}^{\mathrm{T}} \\
\mathbf{x}^{\mathrm{T}}
\end{array}\right]+\left[\begin{array}{l}
\boldsymbol{\eta}_{1}^{\mathrm{T}} \\
\boldsymbol{\eta}_{2}^{\mathrm{T}}
\end{array}\right]+\left[\begin{array}{l}
\boldsymbol{\varepsilon}_{1}^{\mathrm{T}} \\
\boldsymbol{\varepsilon}_{2}^{\mathrm{T}}
\end{array}\right] .
$$

There are many terms in this equation, and we define and explain them in the following paragraphs. First, $\alpha_{1}$ and $\beta_{1}$ are a constant and linear regression coefficient for variable $Y_{1}$, and $\alpha_{2}$ and $\beta_{2}$ are corresponding coefficients for $Y_{2}$. These are known as fixed effects in the LMM. The terms $\boldsymbol{\eta}_{1}$ and $\boldsymbol{\eta}_{2}$ are spatially correlated random effects of mean zero, which we assume conform to a linear model of coregionalization (LMCR, Journel and Huijbregts, 1978). Let us denote some element of $\boldsymbol{\eta}_{i}$, where $i \in\{1,2\}$ by $\eta_{i}(\mathbf{s})$ where $\mathbf{s}$ is a vector with the coordinates of the observation in space. Under the LMCR the covariance of any two observations separated spatially by a lag vector $\mathbf{h}: \eta_{i}(\mathbf{x}), \eta_{i}(\mathbf{x}+\mathbf{h})$, is assumed to depend only on the lag vector and is given by

$$
K_{i, j}(\mathbf{h})=\sum_{k=1}^{s} c_{k}^{i, j} \rho_{k}(\mathbf{h})
$$

where there are $s \geq 1$ independent additive components in the model, the terms $c_{k}^{i, j}$ are variances and covariances that constitute a positive-definite covariance matrix for any $k$ and $\rho_{k}(\mathbf{h})$ is a spatial correlation function. In this study we assume that the correlation function is isotropic (it depends on the lag distance, $|\mathbf{h}|$, not the direction), and can be described by the function due to Matérn (Stein, 1999):

$$
\rho(\mathbf{h})=\frac{1}{2^{\nu-1} \Gamma(\nu)}\left(\frac{2 \nu^{\frac{1}{2}}|\mathbf{h}|}{\phi}\right)^{\nu} \mathcal{K}_{\nu}\left(\frac{2 \nu^{\frac{1}{2}}|\mathbf{h}|}{\phi}\right),
$$

where $\phi$ is a distance parameter, $\nu$ is a smoothness parameter and $\mathcal{K}_{\nu}$ is a modified 
Bessel function of the second kind of order $\nu$. In this study we consider only one spatially correlated component in the LMCR.

Under this model, and the assumption of a normal distribution, the terms $\boldsymbol{\eta}_{1}$ and $\boldsymbol{\eta}_{2}$ are therefore entirely characterized by the variances and covariance $c_{1}^{1,1}, c_{1}^{2,2}$ and $c_{1}^{2,1}$ and the parameters $\phi$ and $\nu$ of the Matérn correlation function.

Each term $\varepsilon_{1}$ and $\varepsilon_{2}$ in Eq. (3) is a zero-mean independently and identically distributed random effect, although there may be a non-zero cross-correlation. Under the assumption of normality, these terms are entirely characterized by their variances and covariance, which we denote by $c_{0}^{1,1}, c_{0}^{2,2}$ and $c_{0}^{2,1}$. These terms are spatially uncorrelated, and so represent components of the variation of our variables which are either not spatially-dependent, or which are spatially dependent at scales too fine to be resolved by the sampling of the variables. This component of the LMM is called the 'nugget', a term inherited from the origin of geostatistics in mining geology.

If the parameters of the LMM are known, then we may use them to predict values of the variables $Y_{1}$ and $Y_{2}$ at unsampled sites where the covariate $X$ is known. There are two components to the prediction. The first may be thought of as a regression-type prediction, depending on the value of the covariate and the fixed effects parameters $\alpha_{1}, \alpha_{2}, \beta_{1}$ and $\beta_{2}$. The second component is a cokriging-type prediction of the random effects $\boldsymbol{\eta}_{1}$ and $\boldsymbol{\eta}_{2}$, and depends on the parameters of the LMCR. The predictions of $Y_{1}$ and $Y_{2}$ have unknown errors at any site. The variance and covariance of these errors can be computed from the LMM.

\subsubsection{Estimation of model parameters and model selection}

In practice, the parameters of the LMM are unknown and must be estimated from data. Under the assumption of normally distributed random effects this can be done by maximum likelihood (ML), although this results in biased estimates of the parameters of the random effects because the fixed effects coefficients are nuisance parameters. An alternative is to use residual maximum likelihood (REML) due to Patterson and Thompson (1971) which is ML estimation applied to a projection of 
the data in which the fixed effects have known zero mean. Once REML estimates of the random effects parameters are obtained then the fixed effects parameters can be estimated by weighted least squares. Marchant and Lark (2007) describe the use of REML to estimate LMCR parameters. REML reduces bias, and so we use it here to estimate model parameters to use in prediction.

One may use ML or REML followed by generalized least squares to estimate the random and fixed effects coefficients of any proposed LMM. In many contexts, including this study, we need to evaluate the evidence for including particular fixed effects. One might ask, for example, whether a model with bathymetry and backscatter as fixed effects is to be preferred to one with a constant mean as the only fixed effect. Equally one might ask whether adding backscatter to a model which includes bathymetry is justified by the available data. These decisions are based on the evidence provided by the maximized likelihood for the fitted models. However, two important points must be noted.

First, a pair of models with different fixed effects (e.g., a model with no covariates so that the overall mean is the only fixed effect, and a model with acoustic backscatter) can be compared on the likelihood, but not on the residual likelihood. For purposes of selecting a fixed effects structure we therefore fitted models by ML, and then used REML to estimate the parameters of the selected model.

Second, adding fixed effects to a model cannot result in a reduction in the maximized likelihood (since, at worst, the coefficient of the new fixed effect can go to zero, leaving the likelihood unchanged). Usually the likelihood is increased by adding new fixed effects. This does not mean that the additional covariates are genuinely informative. One standard criterion used to assess whether additional covariates in a model are justified in terms of improved predictive value is Akaike's information criterion (AIC) (Akaike, 1973). If $\ell$ is the natural logarithm of the maximized likelihood for the fit of a model with $P$ predictors then the AIC is computed as

$$
A=2 P-2 \ell .
$$


One selects from a set of models the one for which AIC is smallest. The criterion can therefore be thought of as based on the goodness of fit, with a penalty for model complexity. By selecting from a set of models the one with the smallest AIC one minimizes the expected information loss through the selection decision (Verbeke and Molenberghs, 2000).

\subsubsection{Prediction from the model}

Estimated parameters of the LMM can be used to obtain predictions at unsampled sites. This prediction is called the empirical best linear unbiased prediction (E-BLUP) (Stein, 1999). Along with the E-BLUP one can also obtain its covariance matrix, the variances and covariance of the prediction errors. For more details of the estimation and prediction procedure, the reader is referred to Marchant and Lark (2007). The E-BLUP and its covariance matrix together define the prediction distribution of the modelled variables at the unsampled site. The prediction distribution represents the uncertainty about the actual values of these variables at that site.

Given the E-BLUP and its covariance matrix at any location, one can simulate values of ALR-transformed gravel, sand and mud content from the prediction distribution. Any simulated set of values can be back-transformed to the original compositional variate by Eq. (2). One may use this sample to estimate properties of the unknown composition at the prediction site, such as the distribution of one of its components. This was done by Lark et al. (2012) in the ordinary cokriging case.

\subsection{Geological setting}

The study area is located approximately $11 \mathrm{~km}$ off the Northumberland coast in northeast England and covers an area of approximately $705 \mathrm{~km}^{2}$ (Fig. 2) with depth of water varying between 50 and $110 \mathrm{~m}$. It was chosen because it was expected to show variability in seabed geomorphology, bathymetry, and associated seabed sediment distribution, providing a suitable environment to test the geostatistical modelling. This variability in seabed character is a function of the complex geological history preserved at seabed including the presence of bedrock at or near-seabed, 
glacial landforms, and mobile sediment bedforms. The high-relief bathymetry also allows for distinct hydrodynamic conditions, as where local bathymetric deeps preserve mud accumulations.

Streamlined glacial landforms, overlying the bedrock, are prominent at the seabed of the study area and reflect ice stream pathways associated with the British ice sheet (BIS) over the last glacial cycle (Clark et al., 2012) (see the bathymetry of the area in Fig. 3a). While our understanding of the extent, timing and dynamics of the glaciation within this sector of the North Sea basin is only recently being improved (Graham et al., 2011; Stewart et al., 2013), the survey area lies just offshore of the projected flow path of the well-described onshore 'Tweed ice stream' (e.g., Everest et al., 2005), and south of the impinging Forth ice stream (Stewart et al., 2013). These streamlined features are interpreted as subglacial landforms elongated parallel to the flow of a palaeo-ice stream (e.g., Stokes and Clark, 2001), and are expected to comprise subglacial till with notable coarse and overconsolidated clay sediment fractions (Gatliff et al., 1994; Stokes et al., 2011). Following the last glacial maximum, marine transgression and flooding of the North Sea brought the re-establishment of marinebased erosional and depositional processes. Across the survey we observe series of sinuous, sharp-crested waves interpreted as mobile sediment bedforms. These modern, frequently sand-dominated features are common in the North Sea, and reflect local hydrodynamic conditions and sediment availability (Huntley et al., 1993).

\subsection{Data}

The bathymetry data were acquired in February-March 2012 in accordance with specifications of the International Hydrographic Organization (2008) for Order 1a, using a Reson 7125 multibeam echosounder aboard the contracted survey vessel $M V$ Neptune. All depth soundings acquired by the multibeam have been reduced to chart datum using a vertical offshore reference frame (VORF) offset value for the centre of the site. The backscatter data were processed with Geocoder software as implemented in Caris HIPS \& SIPS. The bathymetry and backscatter values are 
shown in Fig. 3a and 3b respectively.

The sediment samples were acquired by the RV Cefas Endeavour over March, 2012 with a 0.1-m² Hamon grab, concurrent with the geophysical survey. Sample locations were pre-determined according to anticipated distribution of broadscale habitats based on legacy British Geological Survey (BGS) seabed sediment maps. Discrete samples from 75 stations were collected within the region where swath bathymetry and backscatter data had been acquired. The samples were collected at a variable spacing of approximately $3 \mathrm{~km}$ on average, and sub-sampled for PSA. The positions of the grab-samples are superimposed on Fig. 3a. Legacy BGS sediment samples also exist within the survey area but these were not incorporated due to concerns with positional accuracy, as they were acquired prior to the use of satellite Global Positioning Systems.

The bathymetry and backscatter data are accurate to 2-m grid resolution, but for the purposes of this study were sub-sampled to a 50-m grid spacing (the resolution of the point observations was unchanged but the grid was thinned). This was to reduce the processing requirements for mapping. In principle the mapping could be done on the full resolution data, but it was decided to use a coarser grid because of the relatively sparse distribution of grab-samples. Co-registered bathymetry (metres) and backscatter (decibels) values were extracted at each grab sample location to be used as input for the geostatistical model.

\subsection{Analysis}

\subsubsection{Exploratory data analysis and ALR-transformation}

In section 2.1 the ALR transform was introduced in both general and formal terms. As was noted, Pawlowsky-Glahn and Olea (2004) show that the results of cokriging from ALR-transformed values of a compositional variate do not depend on the initial selection of a component of the composition to serve as the denominator of the log ratio. In other words, one might work with the log-ratios of sand to mud and gravel to mud, or the ratios of mud to sand and gravel to sand etc. In this study we 
used the proportion of gravel as the numerator and computed transformed variables that we denote ALR-Sand and ALR-Mud. Exploratory statistics showed that some of the samples had zero gravel content (Table 1). The ALR transform is not defined in this case. As in the previous study by Lark et al. (2012) we imputed non-zero values for compositions with zeroes, following the method of Martín-Fernandéz and Thió-Henestrosa (2006), and setting zero values to a small value (0.005) less than the smallest non-zero value in the data before renormalizing the affected compostion.

\subsubsection{Model fitting and selection}

Because of the skewness of the ALR-transformed variables (Fig. 5) a Box-Cox transformation was incorporated into the LMM. Under the Box-Cox transformation the modelled variable is $z$ where $y$ is the observed variable (ALR-Mud or sand here), and where

$$
\begin{aligned}
z & =\frac{y^{\lambda}-1}{\lambda} \quad \lambda \neq 0, \\
& =\ln (y) \quad \lambda=0 .
\end{aligned}
$$

Note that if $\lambda=1$ the transformation is just a linear offset. The parameter $\lambda$ is estimated by ML along with the other parameters of the LMM as described in section 2.1 .

Linear mixed models of the form of Eq. (3) were first fitted by maximum likelihood with ALR-Sand and ALR-Mud. In the simplest model there was no covariate so, in effect, the first term on the right-hand side of Eq. (3) was reduced to a vector with two constants, the overall mean of ALR-Sand and ALR-Mud, respectively. We considered a model with acoustic bathymetry as the single covariate, and one with acoustic backscatter as the single covariate. We also considered a model with both acoustic variables as covariates. We chose between these alternative models on the basis of the AIC (Eq. 6) as described in section 2.1.3 above.

Having selected among the alternative models fitted by ML, we refitted the selected model by REML. We also used REML to fit the LMM with overall mean the only fixed effects. This is for comparison with a model in which the acoustic 
data are exploited.

\subsubsection{Cross validation}

Prior to mapping classes with the selected model we undertook two linked validation tests. The first is a test of the LMM to ensure that the E-BLUP based on it gave plausible predictions of the ALR-transformed PSA data, and that the prediction distributions gave a valid measure of uncertainty. The second was an evaluation of the predictions of sediment texture class obtained from the E-BLUP.

In the previous study by Lark et al. (2012) we used a witheld subset of the data to evaluate the cokriging predictions. In this study the total number of sites available was too small to justify this procedure. For purposes of comparison of predictions with and without the use of the acoustic data, we used a cross-validation procedure. In cross-validation each data point was excluded from the data in turn and the EBLUP and its covariance matrix at the location of the excluded data point were computed. Each data value can therefore be compared with its E-BLUP computed from the remaining data.

The first analyses on the cross-validation outputs are to validate the fitted model. Each value of ALR-Sand and ALR-Mud in the data set was compared with the crossvalidation prediction. If the observed value of ALR-Sand at location $\mathbf{s}$ is denoted by $y_{\text {sand }}(\mathbf{s})$ and the E-BLUP is $\tilde{Y}_{\text {sand }}(\mathbf{s})$, then the squared prediction error is

$$
\left\{y_{\text {sand }}(\mathbf{s})-\tilde{Y}_{\text {sand }}(\mathbf{s})\right\}^{2}
$$

and the standardized squared prediction error is

$$
\frac{\left\{y_{\text {sand }}(\mathbf{s})-\tilde{Y}_{\text {sand }}(\mathbf{s})\right\}^{2}}{\sigma_{\text {sand }}^{2}(\mathbf{s})},
$$

where $\sigma_{\text {sand }}^{2}(\mathbf{s})$ is the prediction error variance of ALR-Sand at location s. This is an element of the covariance matrix of the E-BLUP, referred to above. Since the prediction error variance is the expected squared error of the prediction, the expected value of the standardized squared prediction error is 1.0. The median of the standardized squared prediction errors is a diagnostic statistic for the LMM. 
If the parameters of the random effects are correct, and the prediction errors are normal, then the expected median is 0.455 .

The second set of analyses were to evaluate the predictions of sediment texture class. We are interested in mapping the four broad sediment texture classes of the EUNIS habitat classification, 'Coarse', 'Mixed', 'Mud and Sandy Mud', 'Sand and Muddy Sand', shown on the ternary diagram in Fig. 1. These classes underpin marine habitat mapping (Long, 2006). Lark et al. (2012) showed how this could be done with the ordinary cokriging predictions of ALR-transformed particle size data by sampling from the prediction distribution at prediction sites. This is done by using a random number generator to generate a random pair of values for ALRSand and ALR-Mud with means equal to the corresponding E-BLUP and covariance matrix equal to the E-BLUP error covariance matrix. The pair of simulated values is then back-transformed to the simplex space of the sand-mud-gravel composition and the corresponding texture class is identified. By repeating this many times one may estimate the probability of each class. This was done for each cross-validation site. If a total of $N$ samples is drawn from the probability distribution for location $\mathbf{s}$, and $n_{i}(\mathbf{s})$ of the samples correspond to class $i$, then the estimated probability that the $i$ th class occurs at location $\mathbf{s}$ is

$$
\widehat{p}_{i}(\mathbf{s})=\frac{n_{i}(\mathbf{s})}{N} .
$$

At site $\mathbf{s}$ the best prediction of the texture class, in the sense of the prediction most likely to be correct, is the class for which the estimated probability, $\widehat{p}_{i}(\mathbf{s})$, is largest. We evaluated these predictions over all sites. Over all sites the known class may be compared with the most probable class (the class to which most of the samples from the prediction distribution belong) in an error matrix. Various measures of the success of the prediction can be computed from this matrix (Lark, 1995). The first is the overall purity of the mapped legend classes, estimated by the proportion of cross-validation sites in which the observed class is also the most probable class. For each class, one may compute a separate purity, estimated by 
the proportion of those cross-validation sites at which the class of interest is the most probable where this class is the one actually observed. One may also compute the representation of each class. The representation of a class is estimated by the proportion of those cross-validation sites where that class is observed at which it is the most probable class according to the sample from the prediction distribution.

The overall purity of the prediction should be compared with two benchmark values. One is the proportion of all sites in the most frequent class (since that is the overall purity of a notional map in which all sites are allocated to the most frequent class). The second is the probability of correctly identifying the class at a site by a random guess where the probability of guessing class $i$ is equal to the overall proportion of the area of interest in class $i$. We estimated the probability of guessing each class from the proportion of the sample data in that class. Let $\widehat{\pi}_{i}$ be the proportion of the sample in the $i$ th out of $I$ classes. The estimate of the probability of correctly identifying the class by a random guess is then

$$
\sum_{i=1}^{I} \widehat{\pi}_{i}^{2} .
$$

The overall purity, class purity and class representations are based only on the most-probable mapped class at each validation location. This is the best prediction at a site, but the output of the procedure presented in this paper also gives an indication of how confident we can be in the prediction because probabilities are computed for all classes. Consider two locations at which the probabilities for the four sediment classes are, respectively, $\{0.5,0.2 .0 .25,0.05\}$ and $\{0.8,0.1 .0 .05,0.05\}$. In both cases the first class is the most probable, but we are more certain about the allocation in the second case. In the context of this study we are interested in whether the confidence in the predicted texture class, as measured by the class probabilities, is improved by including bathymetry and backscatter in the LMM. Two such sets of probabilities can be compared by computing their respective entropies. If $\pi_{i}$ is the probability of the $i$ th out of $m$ classes at some location, then the entropy 
of the set of probabilities over all classes is:

$$
\mathcal{E}=-\sum_{i=1}^{m} E_{i}
$$

where

$$
\begin{aligned}
E_{i} & =\pi_{i} \log \pi_{i}, & & \pi_{i}>0 \\
& =0 . & & \pi_{i}=0
\end{aligned}
$$

If natural logarithms are used then the entropy is measured in nats. The entropy for the two example sets of probabilities given above are 1.16 and 0.71 nats respectively. The entropy is larger for the more uniform ensemble of probabilities where the uncertainty is larger. We call the entropy of the set of probabilities, computed from the E-BLUP prediction distribution at a site, the prediction entropy at that site. As a measure of the uncertainty of the predictions with and without the bathymetry and backscatter data, we computed the mean prediction entropy for each method over the cross-validation points. We also computed the entropy for the overall proportions of all the classes. The comparison between this class entropy and the mean prediction entropy is a measure of how much additional information the E-BLUP provides.

Note that the mean entropy of a set of class predictions is computed without reference to whether the most-probable class is the observed class at these sites. It is therefore a measure of the 'internal' confidence of the prediction, i.e., the extent to which the computed class probabilities indicate one very likely class at that site, or several classes with similar probabilities. In general one might expect map purities and representations to be improved by the inclusion of a covariate in the LMM which reduces the mean entropy, but in principle these two sets of statistics could show opposing trends. It is therefore necessary to compute both sets of statistics for a full evaluation of a predictive model and to compare them both between models.

\subsubsection{Mapping}

The prediction distribution from the E-BLUP was evaluated at each node of 
the bathymetry/backscatter 50-m grid using the LMM with no covariates and the LMM with covariates selected from bathymetry and backscatter according to AIC, as described in the previous section. By numerical integration, the probability of each EUNIS sediment class was evaluated at each node. On this basis the most probable class could be identified and mapped, as could the probability of any one class. The entropy of the set of probabilities at each node was evaluated using Eq. 12.

\section{Results}

\subsection{Exploratory statistics}

Table 1 shows summary statistics of the data from PSA expressed as percentages. Fig. 4 shows the ALR-transformed sand and mud data (with gravel as the denominator) expressed as quartiles (divisions of the range of values which partition the data into four equal-sized subsets). The figure shows the spatial dependence of the PSA data; neighbouring observations tend to be more similar with respect to both variables than observations which are further apart in space. This is encouraging because such spatial dependence is captured by the LMM and provides the basis for the spatial prediction. In Fig. 5 the histograms of the ALR-Sand and mud contents are shown, and scatter plots of these two variables and of the bathymetry and backscatter values at the grab-sample locations. Note that both ALR-Sand and ALR-Mud have skew distributions, despite the log-transformations. This is the rationale for the inclusion of the Box-Cox transformation in the further analysis (Eq. 7). ALR-Mud and ALR-Sand are strongly correlated. Because this correlation appears after the ALR transform we can be confident that it is not a spurious correlation due to compositional effects (Pawlowsky-Glahn and Olea, 2004). It appears that, in this particular setting, the sandy and muddy sediments have comparable spatial distributions relative to gravel. This can also be seen in Fig. 4, the spatial distribution of the quartiles of ALR-Mud and ALR-Sand are very similar.

The bathymetry and backscatter values also show a strong correlation, which 
can also be seen in Figs. 2 and 3, the backscatter is smallest from deeper locations. This correlation is of interest because it is possible that one of these variables could be regarded as a proxy for the other, and that adding both to the LMM is therefore not justified. This is tested by the use of AIC for model selection.

In Fig. 6 are shown scatter-plots of the ALR-transformed sand and mud content against both backscatter and bathymetry. The correlation coefficients are also shown on each plot. Note that there is a moderate to strong correlation between both ALRMud and ALR-Sand and backscatter, and a moderate correlation in both cases with bathymetry. This is encouraging because it suggests that either or both covariates may provide useful information for statistical prediction of the sediment data as fixed effects in the LMM. Note that there is more scatter in these plots where the backscatter is small (deeper sites). This potentially introduces some kurtosis (heavy tails) into the distribution of the random component of the LMM, which is another advantage of including the Box-Cox transformation.

\subsection{Model-fitting}

Table 2 presents the statistics of the LMMs. Note that the AIC is smallest for the model with bathymetry and backscatter both included as fixed effects. This is interesting because it shows that both covariates together provided more information about sediment composition than did either covariate separately, despite their strong correlation. Because the Box-Cox transformation differs between the models, we may not compare their variance components directly. However, it is interesting to note that in the model with no covariates, the correlated variances and covariances in the model $\left(c_{1}^{\mathrm{S}, \mathrm{S}}, c_{1}^{\mathrm{M}, \mathrm{M}}\right.$ and $\left.c_{1}^{\mathrm{S}, \mathrm{M}}\right)$ are all notably larger than the corresponding nugget variances and covariances $\left(c_{0}^{\mathrm{S}, \mathrm{S}}, c_{0}^{\mathrm{M}, \mathrm{M}}\right.$ and $\left.c_{0}^{\mathrm{S}, \mathrm{M}}\right)$. However, in the model with bathymetry and backscatter included as covariates, the position is reversed, and the nugget components of variance are the largest. This indicates that much of the long-range spatial variability that the relatively sparse grab-sampling resolves is accounted for by the covariates. 
One may also compute the correlation between the coregionalized random components for ALR-Sand and ALR-Mud in the LMM. In this case there are two components, one of which is the nugget and the other the spatially correlated component. In the case of the model with no covariates this 'structural correlation' is 0.98 for the nugget component and 0.96 for the spatially correlated component of the model, showing that these particle size components are similarly strongly correlated at both fine and coarse spatial scales. By contrast, the nugget and spatially correlated structural correlations for the random terms, in the model with bathymetry and backscatter included as covariates, are 0.96 and 0.75 respectively. This shows that the fine-scale variability in ALR mud and sand not accounted for by the covariates remain strongly correlated, but that the coarser-scale variation is somewhat less strongly correlated. In other words, the covariates account for coarse-scale sources of variation which affect both mud and sand content of the sediment similarly, and the patterns of coarse-scale variation which remains unaccounted for by these covariates are similar for the two variables, but rather less similar than the corresponding fine-scale variation.

Figure 7 shows the histograms of the cross-validation error for both variables under both LMM. In all cases, the errors have a symmetrical distribution which resembles a normal distribution. Table 3 shows the cross-validation results for BoxCox transformed ALR-Sand and mud content. The mean square error for both ALR-Sand and ALR-Mud (Eq. 8) is notably reduced by including bathymetry and backscatter as covariates. Note that in all cases the mean standardized squared prediction error (Eq. 9) is close to one. Recall from section 2.4.3 that this statistic measures how well the prediction distribution of the E-BLUP characterizes its uncertainty. The mean standardized squared prediction error should be close to one, this is effectively a check on the model-fitting procedure. More sensitive as a test of the fitted LMM are the median values, expected to be close to 0.455 for normallydistributed prediction errors. In all cases the values fall within the $95 \%$ confidence 
interval for the sample median with a sample of his size, $[0.22,0.64]$, suggesting that the model is reliable. In both cases, the median values are somewhat closer to the expected value for the model with both covariates included. One possible reason for this is that the covariates account for outlying values in the data, either marginal outliers (particularly large or small values) or spatial outliers (values that appear unusual in spatial context).

Tables $4 \mathrm{a}$ and $4 \mathrm{~b}$ show the results from computing probabilities for each EUNIS sediment class from the cross-validation distributions. The first part of each table shows the comparison between the most-probable class and the observed class, and the associated representations and purities. The overall proportion of validation sites where the most probable class, inferred from the E-BLUP distribution corresponds to the observed class is 0.58 when the only fixed effect is a constant, and is increased to 0.65 by inclusion of bathymetry and backscatter as fixed effects. These proportions may be compared with 0.47 (the proportion of sites in the most common class) and 0.31 (the probability of correctly allocating the class by guesswork; Eq.11). Note that all class purities are improved by including bathymetry and backscatter (apart from class 'Mixed' which is unaffected) and the representation of all classes apart from Sand and Muddy Sand is also improved. The overall class entropy is 1.27 nats. This is reduced to 1.13 by using the E-BLUP with no covariates, and to 0.96 by the inclusion of bathymetry and backscatter as covariates.

\subsection{Mapping}

Figure 8 shows the class of maximum probability according to the E-BLUP with (8a) the constant the only fixed effect and (8b) bathymetry and backscatter included as fixed effects. Given the relatively sparse sampling it is not surprising that the map based only on the sample data is more generalized than the map incorporating the backscatter and bathymetry. In particular, the Mixed class is more restricted in its distribution across the area. Both maps reveal the effect of broad-scale bathymetry (Fig. 2) on the distribution of seabed sediment. Coarser 
sediment fractions (including 'coarse' and 'mixed') are typically associated with the bathymetric platforms within the area, and the bathymetric deep in the southeast of the study area shows a clear association with mud at seabed. These broad patterns can be seen in the map in Fig. 8a, which is based on the LMM where the only fixed effect is a constant and bathymetry is not used for prediction. They are, of course, more apparent in the map in Fig. 8b, based on the LMM which incorporates the acoustic data.

Sediment class predictions based on the acoustic data also suggest clearer affinities between smaller-scale seabed geomorphology and sediment distribution (Fig. $8 \mathrm{~b})$. This result is consistent with our understanding of the geology of the region e.g., glacially streamlined landforms (NW-SE oriented) are more likely to comprise coarse sediments (e.g., Colgan et al., 2005). This level of detail is not captured by the map presented in Fig. 8a where the acoustic data are not fixed effects in the LMM. Fig. 8c shows some of the predictions from the model with acoustic data at larger scale. This shows the coarser texture of sediment on glacially streamlined landforms (NW-SE orientation) captured by the model prediction where the features are sufficiently large (e.g., between 'Mixed' vs. 'Sand' classes). Note that there are significant variations of landform in Fig. 8c within the area where 'Mud' is the most probable class.

Figure 9 shows the probability of the most probable class. Again, the spatial pattern is more general in Fig. 9a with no covariates, and the distribution of sample points dominates the pattern. Note, in both maps, the areas with greater uncertainty in two large areas where 'Coarse' is the most probable class and one in the west of the region where Mud and Sandy Mud is most probable. Not surprisingly, there is greater uncertainty at or near the boundaries between adjacent sediment classes. The vertical striping in Fig. (9b) reveals the persistence of artefacts in the acoustic data through to the modelled outputs. Here the striping artefact mimics the survey track lines, where the outer beams of the acoustic backscatter data are not entirely 
normalized with those from adjacent lines. This emphasizes the requirement for high quality data acquisition and processing protocols when employing these methods.

As discussed previously, the information in the local prediction distribution of the transformed compositional variables can be presented in terms of the most probable class, and its probability (as in Figs. 8 and 9) but it is also possible to show the probability of occurrence of particular sediment texture classes. For example, Fig. 10 shows the probability of occurrence of texture class 'Coarse'. The two maps show broadly similar spatial patterns, with finer spatial resolution where the acoustic covariates are included.

As discussed in section 2.4.3., the degree of uncertainty in any local prediction distribution, with respect to the probability of the sediment texture classes, is summarized by the classification entropy which is large when the probabilities of each of the set of classes are similar (so uncertainty is greatest). Over $80 \%$ of the region the entropy is smaller when the covariates are used (Fig. 11), i.e., the uncertainty about the classes is reduced by incorporating the acoustic information.

\section{Discussion and Conclusions}

Our results show that the predictive accuracy of geostatistical mapping of sediment texture classes can be improved by incorporation of bathymetry and backscatter data. This can be achieved by treating these variables as covariates in the LMM. The prediction is improved as judged from the cross validation error matrices and associated summary statistics, and also by the prediction entropy which shows that the uncertainty is, on average, reduced by the additional information. In contrast to the predictions from the model with a constant mean as the only fixed effect, model predictions incorporating the acoustic data captured finer detail in the distribution of sediments. These patterns are geologically plausible, reflecting the effects of several environmental processes which act upon the seabed at varying spatial and temporal scales (e.g., sedimentation of finer sediments in lower energy deeps; seabed exposure of coarser sediment associated with glacial landforms). 
We have demonstrated the value of the LMM and E-BLUP for predicting sediment texture properties from grab samples and bathymetry and backscatter data in one study area. Further studies are needed to evaluate the method over a wider range of conditions. It is known from previous studies, cited above, that sediment properties are correlated with bathymetry and acoustic backscatter. Furthermore, since sediment properties depend on processes that operate at different spatial scales it is very likely that they will generally show spatial dependence. Given these two considerations it is reasonable to expect that the LMM will be found to be a generally useful model for this purpose.

This approach retains the advantages of the ordinary cokriging approach presented by Lark et al. (2012), whilst allowing the additional information in the covariates to be exploited. The advantages are that the linear mixed modelling is a robust and reproducible process, with internal model validation (through crossvalidation on the prediction distributions). It is an objective process, not requiring expert interpretation, which may make it rather more rapid and less costly than conventional geological mapping. Finally, unlike data mining methods (such as machine learning), the LMM is a formal statistical model. The prediction distributions that are produced from it allow probabilistic assessments to be made of the uncertain allocations of locations to sediment classes, and can also be interrogated directly to provide estimates of the actual sand, mud and gravel content where this is required. The covariates, being more densely sampled than the direct observations of sediment, allow more accurate predictions to be made at sites across the study area.

Conventional geological mapping of sediments is laborious and requires experienced staff. However, process-based interpretation of observations provides a sound basis for mapping. In circumstances where an existing sediment map has been produced by geological interpretation, and new data become available (e.g., sediment data and bathymetry and backscatter), then the LMM could be used to update the map by incorporating its units as a fixed effect. In this case the different mapped 
units become (categorical) fixed effects in the LMM, along with the continuous covariates (bathymetry and backscatter).

While, as noted above, the LMM is a formal probabilistic model unlike data mining methods, the latter can be particularly useful for dealing with non-linear relationships between target variables and predictors. It would therefore be useful to compare the LMM with data mining methods, and expert interpretation, in a range of settings. Such comparative studies have been rare to date, but the one by Diesing et al. (2014) demonstrates their potential value. It would be useful to carry out such studies in a range of conditions to compare the LMM with expert intepretation and data mining methods with respect to both statistical measures of prediction quality and the time and cost requirements of the methods.

It was notable that incorporating the covariates into the predictive models had the largest effect on the spatially correlated residual variance; i.e., the covariates largely explained the broader-scale patterns of variation in sediment composition. In the models with covariates included the nugget variance, i.e., the variance not resolved by sampling, was larger than the correlated variance. The fine-scale variation in the ALR-mud and ALR-sand were strongly correlated, suggesting that they reflect common processes that these covariates did not capture. As a result, the final map gives a good impression of the general trends in sediment composition, but predicted variations over short distances are less certain.

The structure of the LMM lends itself to the incorporation of additional information. For example, geomorphological interpretation of bathymetry, or continuous predictors based on the bathymetric surface (rugosity, slope etc.) might be incorporated as covariates. Hydrodynamic model outputs (e.g., current speeds and wave orbital velocity) might similarly be incorporated as predictors, and the log-ratio testing procedure used here can be applied to evaluate the evidence for particular sources of information before a particular model is used for prediction. Finally, one possible way to reduce the residual variance of the model at the finest scales might be 
through physical modelling of the characteristics of the backscatter (e.g., Sternlicht and de Moustier, 2003).

In conclusion, the LMM was shown to be an effective method to incorporate backscatter and bathymetry information into the prediction of sediment texture classes. There is scope to use this approach to incorporate a wider range of predictors, including outputs from physical models or other variables derived from bathymetry. It could also be used to incorporate conventional geological maps of the seabed as a way to update these with new data. There is scope for further work to develop this method (particularly for upscaling predictions) and to compare it with other approaches.

\section{Acknowledgements}

The contributions of RML, BPM, DD, SLG and HS are published with the permission of the Executive Director of the British Geological Survey (NERC), and this work was supported by NERC's Marine Environmental Mapping (MAREMAP) programme (http://www.maremap.ac.uk). Multibeam echosounder and grab sampling data were collected as part of the 'Coordination of the Defra Marine Conservation Zone data collection programme 2011/12' funded by the Department of Environment, Food and Rural Affairs of the United Kingdom. 


\section{References}

Aitchison, J., 1986. The Statistical Analysis of Compositional Data. Chapman and Hall, London.

Akaike, H., 1973. Information theory and an extension of the maximum likelihood principle. In: Petov, B.N., Csaki, F. (Eds.), Second International Symposium on Information Theory. Akademia Kiado, Budapest, pp. 267-281.

Anderson, J.T., Gregory, R.S., Collins, W.T., 2002. Acoustic classification of marine habitats in Newfoundland. ICES Journal of Marine Science 59, 156-167.

Bale, A.J., Kenny, A.J., 2005. Sediment analysis and seabed characterization. In: N.A. Holme, N.A., McIntyre, A.D. (Eds.), Methods for the study of marine benthos. Blackwell, Oxford, pp. 43-86.

Brown, C.J., Smith, S.J., Lawton, P., Anderson, J.A.T., 2011. Benthic habitat mapping: A review of progress towards improved understanding of the spatial ecology of the seafloor using acoustic techniques. Estuarine, Coastal and Shelf Science 92, 502-520.

Burgess, T.M., Webster, R., 1980. Optimal interpolation and isarithmic mapping of soil properties. II Block kriging. Journal of Soil Science 31, 333-341.

Clark, C.D., Hughes, A.L.C., Greenwood, S.L., Jordan, C.J., Sejrup, H.P., 2012. Pattern and timing of retreat of the last British-Irish Ice Sheet. Quaternary Science Reviews 44, 112-146.

Colgan, P.M., Kickelson, D.M., Cutler, P.M., 2005. Ice-marginal terrestrial landsystems: southern Laurentide ice sheet margin. In: Evans, D.J.A. (Ed.), Glacial Landsystems. Hodder Arnold, London, pp. 111-142.

Connor, D.W., Gilliland, P.M., Golding, N., Robinson, P., Todd, D., Verling, E., 2006. UKSeaMap: the mapping of seabed and water column features of UK Seas. Joint Nature Conservation Committee, Peterborough. 
Dartnell, P., Gardner, J.V., 2004. Predicting seafloor facies from multibeam bathymetry and backscatter data. Photogrammetric Engineering and Remote Sensing 70, $1081-1091$.

Diesing, M., Green, S.L., Stephens, D., Lark, R.M., Stewart, H.A., Dove, D., 2014. Mapping seabed sediments: Comparison of manual, geostatistical, objectbased image analysis and machine learning approaches. Continental Shelf Research 84, 107-119.

Endler, M., Endler, R., Bobertz, B., Leipe, T., Arz, H.W., 2015. Linkage between acoustic parameters and seabed sediment properties in the south-western Baltic Sea. Geo-Marine Letters 35, 145-160.

Evans, J.L., Peckett, F., Howell, K.L., 2015. Combined application of biophysical habitat mapping and systematic conservation planning to assess efficiency of representativeness of the existing High Seas MPA network in the Northeast Atlantic. ICES Journal of Marine Science 72, 1483-1497.

Everest, J., Bradwell, T., Golledge, N., 2005. Subglacial landforms of the Tweed palaeo-ice stream. The Scottish Geographical Magazine 121, 163-173.

Folk, R.L., 1954. The distinction between grain size and mineral composition in sedimentary rock nomenclature. Journal of Geology 62, 344-359.

Fonseca, L., Brown, C., Calder, B., Mayer, L., Rzhanov, Y., 2009. Angular range analysis of acoustic themes from Stanton Banks Ireland: A link between visual interpretation and multibeam echosounder angular signatures. Applied Acoustics 70, 1298-1304.

Gatliff, R. W., Richards, P.C., Smith, K., Graham, C.C., McCormac, M., Smith, N. J. P., Long D., Cameron, T.D.J., Evans, D., Stevenson, A.G., Bulat, J., Ritchie, J.D., 1994. United Kingdom offshore regional report: the geology of the central North Sea. British Geological Survey, HMSO, London. 
Glynn, B., Brown, C., Rooney, S., 2008. Coincident swath acoustic backscatter and bathymetry for the interpretation of shallow-water sediment composition and processes. Journal of the Geological Society 165, 597-607.

Goff, J.A., Kraft, B.J., Mayer, L.A., Schock, S.G., Sommerfield, C.K., Olson, H.C., Gulick, S.P.S., Nordfjord, S., 2004. Seabed characterization on the New Jersey middle and outer shelf: correlatability and spatial variability of seafloor sediment properties. Marine Geology 209, 47-172.

Graham, A.G.C., Stoker, M.S., Lonergan, L., Bradwell, T., Stewart, M.A., 2011. The Pleistocene glaciations of the North Sea basin. Developments in Quaternary Science 15, 261-278.

Haris, K., Chakraborty, B., 2014. Stochastic formalism-based seafloor feature discrimination using multifractality of time-dependent acoustic backscatter. Nonlinear Processes in Geophysics 21, 101-113.

Howell, K.L., 2010. A benthic classification system to aid in the implementation of marine protected areas networks in the deep/high seas of the NE Atlantic. Biological Conservation 143, 1041-1056.

Hughes Clarke, J.E., Mayer, L.A., Wells, D.E., 1996. Shallow-water imaging multibeam sonars: a new tool for investigating seafloor processes in the coastal zone and on the continental shelf. Marine Geophysical Researches 18, 607-629.

Huntley, D.A., Huthnance, J.M., Collins, M.B., Liu, C-L., Nicholls, R.J., Hewitson, C., Green, M.O., Dyer, K.R., Jago, C.F., 1993. Hydrodynamics and sediment dynamics of North Sea sand waves and sand banks. Philosophical Transactions of the Royal Society of London. Series A: Physical and Engineering Sciences $343,461-474$.

International Association of Oil \& Gas Producers, 2013. Guidelines for the conduct of offshore drilling hazard site surveys. International Association of Oil \& Gas 
Producers Report No. 373-18-1 http://www.ogp.org.uk/pubs/373-18-1.pdf

International Hydrographic Organization, 2008. IHO standards for hydrographic surveys, $5^{\text {th }}$ Edition, IHO Special Publication S-44, International Hydrographic Bureau, Monaco.

Journel, A.G., Huijbregts, C.J., 1978. Mining Geostatistics. Academic Press, London.

Kostylev, V.E., Todd, B.J., Fader, G.B.J., Courtney, R.C., Cameron, G.D.M., Pickrill, R.A., 2001. Benthic habitat mapping on the Scotian Shelf based on multibeam bathymetry, surficial geology and sea floor photographs. Marine Ecology Progress Series, 219, 121-137.

Lark, R.M., 1995. Components of accuracy of maps with special reference to discriminant analysis on remote sensor data. International Journal of Remote Sensing 16, 1461-1480.

Lark, R.M., Dove, D., Green, S.L., Stevenson, A., Stewart, H., Stevenson, A., 2012. Spatial prediction of seabed sediment texture classes by cokriging from a legacy database of point observations. Sedimentary Geology 281, 35-49.

Long, D., 2006. BGS detailed explanation of seabed sediment modified Folk classification. MESH (Mapping European Seabed Habitats) available at http://www.searchmesh.net/PDF/GMHM_Detailed_explanation_of_seabed_sediment_classification.pd

Lucieer, V., Hill, N.A., Barrett, N.S., Nichol, S., 2013. Do marine substrates 'look' and 'sound' the same? Supervised classification of multibeam acoustic data using autonomous underwater vehicle images. Estuarine, Coastal and Shelf Science 117, 94-106.

Marchant, B.P., Lark, R.M., 2007. Estimating of linear models of coregionalization by residual maximum likelihood. European Journal of Soil Science 58, 15061513. 
Marsh, I., Brown, C., 2009. Neural network classification of multibeam backscatter and banthymetry data from Stanton Bank (Area IV). Applied Acoustics 70, $1269-1276$.

Martín-Fernandéz, J.A., Thió-Henestrosa, S., 2006. Rounded zeros: some practical aspects for compositional data. In: Buccianti, A., Mateu-Figueras, G., Pawlowsky-Glahn, V. (Eds.), Compositional Data Analysis in the Geosciences: From Theory to Practice. Geological Society of London Special Publications 264, pp. 191-201.

McGonigle, C., Collier, J., 2014. Interlinking backscatter, grain size and benthic community structure. Estuarine, Coastal and Shelf Science 147, 123-136.

Patterson, H.D., Thompson, R., 1971. Recovery of inter-block information when block sizes are unequal. Biometrika 58, 545-554.

Pawlowsky-Glahn, V., Olea, R.A., 2004. Geostatistical Analysis of Compositional Data. Oxford University Press, New York.

Snellen, M., Siemes, K., Simons, D., 2011. Model-based sediment classification using single-beam echosounder signal. Journal of the Acoustic Society of America 129, 2878-2888.

Stein, M.L., 1999. Interpolation of spatial data: some theory for kriging. Springer, New York.

Sternlicht, D.D., de Moustier, C.P., 2003. Time dependent seafloor acoustic backscatter $(10-100 \mathrm{kHz})$. Journal of the Acoustic Society of America 114, 2727-2743.

Stokes, C.R., Clark, C.D., 2001. Palaeo-ice streams. Quaternary Science Reviews $20,1437-1457$.

Stokes, C.R., Spagnolo, M., Clark, C.D., 2011. The composition and internal structure of drumlins: Complexity, commonality, and implications for a unifying theory of their formation. Earth-Science Reviews 107, 398-422. 
Verbeke, G., Molenberghs, G., 2000. Linear Mixed Models for Longitudinal Data. Springer-Verlag, New York.

Wentworth, C.K., 1922. A scale of grade and class terms for clastic sediments. The Journal of Geology 30, 377-392. 
Table 1 Summary statistics of particle size class data.

\begin{tabular}{lrrr}
\hline & $\begin{array}{c}\text { Sand } \\
\text { Percent by }\end{array}$ & $\begin{array}{c}\text { Mudavel } \\
\text { Meas }\end{array}$ \\
\hline & & & \\
Median & 72.93 & 14.22 & 12.85 \\
Standard deviation & 12.91 & 7.86 & 12.97 \\
First quartile & 64.02 & 8.3 & 1.07 \\
Third quartile & 79.36 & 20.84 & 21.68 \\
Minimum & 42.58 & 1.13 & 0.00 \\
Maximum & 98.76 & 31.9 & 45.11 \\
Minimum non-zero & 42.58 & 1.13 & 0.018 \\
value & & & \\
\hline
\end{tabular}




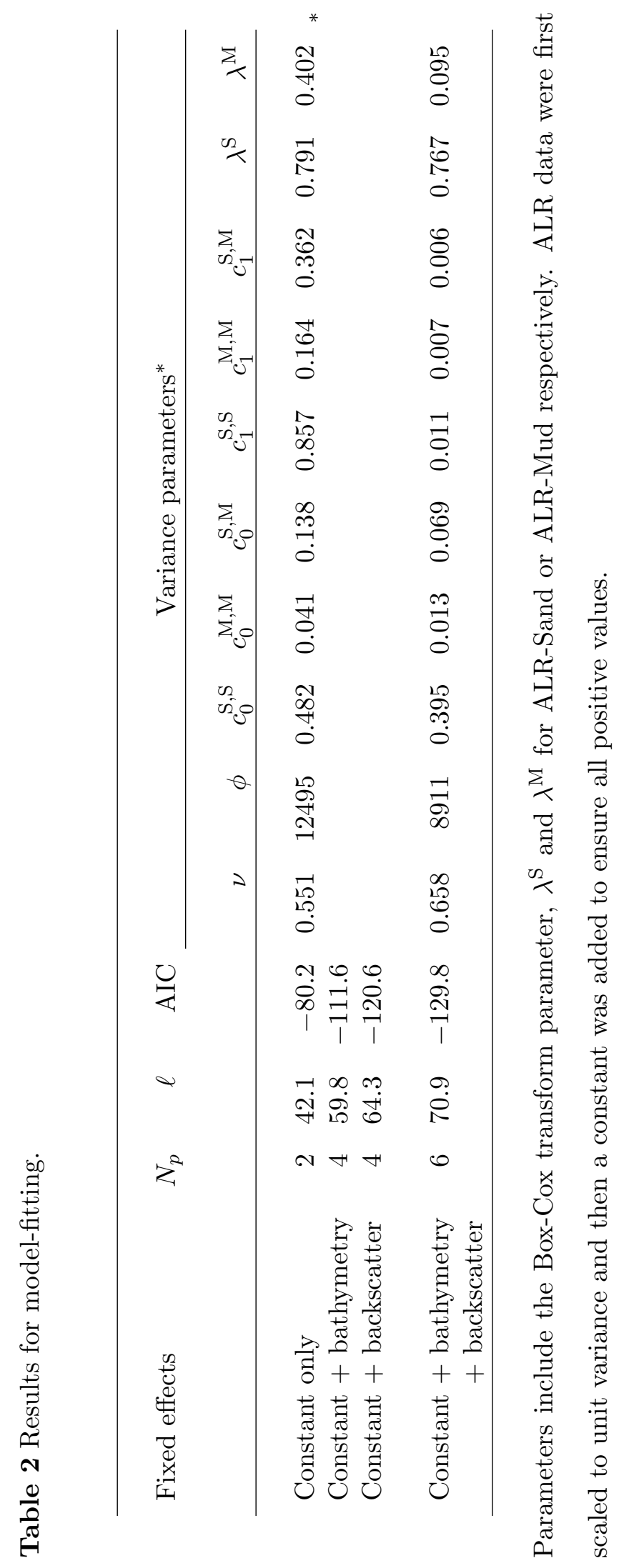


Table 3 Cross-validation results for (Box-Cox transformed) ALR-Sand and ALRMud.

\begin{tabular}{|c|c|c|c|c|c|c|}
\hline \multirow[t]{3}{*}{ Fixed effects } & \multicolumn{2}{|c|}{$\begin{array}{l}\text { Mean square } \\
\text { error }\end{array}$} & \multicolumn{4}{|c|}{$\begin{array}{c}\text { Standardized squared } \\
\text { prediction error }\end{array}$} \\
\hline & & & \multicolumn{2}{|c|}{ Mean } & \multicolumn{2}{|c|}{ Median } \\
\hline & Sand & Mud & Sand & Mud & Sand & Mud \\
\hline Constant only & 0.76 & 0.09 & 1.03 & 1.04 & 0.38 & 0.33 \\
\hline $\begin{aligned} \text { Constant } & + \text { acoustic bathymetry } \\
& + \text { acoustic backscatter }\end{aligned}$ & 0.44 & 0.02 & 1.05 & 1.05 & 0.46 & 0.35 \\
\hline
\end{tabular}


Table 4a Cross-validation results for prediction of sediment texture classes; constant the only fixed effect.

Distribution of observations

\begin{tabular}{|c|c|c|c|c|c|}
\hline \multirow[b]{2}{*}{$\begin{array}{l}\text { Observed } \\
\text { Class }\end{array}$} & \multicolumn{4}{|c|}{ Most probable class } & \multirow[b]{2}{*}{ Representation } \\
\hline & Coarse & Mixed & $\begin{array}{c}\text { Mud \& } \\
\text { Sandy Mud }\end{array}$ & $\begin{array}{c}\text { Sand \& } \\
\text { Muddy Sand }\end{array}$ & \\
\hline Coarse & 8 & 0 & 1 & 3 & 0.66 \\
\hline $\begin{array}{l}\text { Mixed } \\
\text { Mud \& }\end{array}$ & 4 & 22 & 3 & 7 & 0.61 \\
\hline $\begin{array}{l}\text { Sandy Mud } \\
\text { Sand \& }\end{array}$ & 1 & 4 & 8 & 3 & 0.50 \\
\hline Muddy Sand & 3 & 3 & 0 & 7 & 0.54 \\
\hline Purity & 0.50 & 0.76 & 0.66 & 0.35 & \\
\hline
\end{tabular}

Proportion of validation sites

in most-probable class

0.58

Expected proportion correct

by random allocation

0.31

Proportion in commonest class

(Mixed)

Class entropy

Mean prediction entropy

1.13 
Table $4 \mathbf{b}$ Cross-validation results for prediction of sediment texture classes; acoustic bathymetry and backscatter as fixed effects.

Distribution of observations

\begin{tabular}{|c|c|c|c|c|c|}
\hline \multirow[b]{2}{*}{$\begin{array}{l}\text { Observed } \\
\text { Class }\end{array}$} & \multicolumn{4}{|c|}{ Most probable class } & \multirow[b]{2}{*}{ Representation } \\
\hline & Coarse & Mixed & $\begin{array}{c}\text { Mud \& } \\
\text { Sandy Mud }\end{array}$ & $\begin{array}{c}\text { Sand \& } \\
\text { Muddy Sand }\end{array}$ & \\
\hline Coarse & 9 & 2 & 0 & 1 & 0.75 \\
\hline $\begin{array}{l}\text { Mixed } \\
\text { Mud \& }\end{array}$ & 5 & 25 & 2 & 4 & 0.69 \\
\hline $\begin{array}{l}\text { Sandy Mud } \\
\text { Sand \& }\end{array}$ & 0 & 1 & 10 & 5 & 0.63 \\
\hline Muddy Sand & 2 & 5 & 0 & 6 & 0.46 \\
\hline Purity & 0.56 & 0.76 & 0.83 & 0.38 & \\
\hline
\end{tabular}

Proportion of validation sites

in most-probable class

Expected proportion correct

by random allocation

Proportion in commonest class

(Mixed)

Class entropy

Mean prediction entropy

0.96 


\section{Figure Captions}

Figure 1. EUNIS sediment texture classes defined on the ternary diagram for silt, mud and sand.

Figure 2. High-resolution swath bathymetry of the study area with grab sample locations indicated. The location of the study area off the northeast coast of England is shown in inset. Acoustic and ground-truthing data provided courtesy of Defra.

Figure 3. Acoustic backscatter data expressed in decibels (dB) with grab sample locations indicated. Acoustic and ground-truthing data provided courtesy of Defra.

Figure 4. Post-plot of ALR-transformed sand and mud content. The data are divided into four sets defined by the quartiles of the distribution, so 'Q1' indicates observations smaller than the first quartile, i.e., the smallest $25 \%$ of all data.

Figure 5. Histograms of ALR-transformed sand and mud content (top), scatter plots of ALR-transformed sand and mud content and acoustic bathymetry and backscatter with correlation coefficients (R) (bottom).

Figure 6. Scatterplots of ALR-transformed sand and mud content against acoustic backscatter (top) and bathymetry (bottom). Correlation coefficients (R) are also shown.

Figure 7. Histograms of cross-validation kriging errors for Box-Cox transformed values of ALR-Sand and ALR-Mud using model with constant mean the only fixed effect (top) or the selected model with both acoustic bathymetry and backscatter as fixed effects (bottom).

Figure 8. Class of maximum probability according to E-BLUP distribution with (a) constant only fixed effect, (b) bathymetry and backscatter as fixed effects, 
(c) a section of the map obtained using bathymetry and backscatter in shown at larger scale. The rectangle on (b) indicates the area of (c). Sediment class predictions are overlain on shaded-relief backscatter.

Figure 9. Probability of class of maximum probability according to E-BLUP distribution with (a) constant only fixed effect, and (b) bathymetry and backscatter as fixed effects.

Figure 10. Probability of class 'Coarse' according to E-BLUP distribution with (a) constant only fixed effect, and (b) bathymetry and backscatter as fixed effects. Values are overlain on shaded-relief bathymetry to demonstrate association between geomorphology and seabed sediment distribution.

Figure 11. Entropy of class prediction probabilities according to E-BLUP distribution with (a) constant only fixed effect and (b) bathymetry and backscatter as fixed effects. 


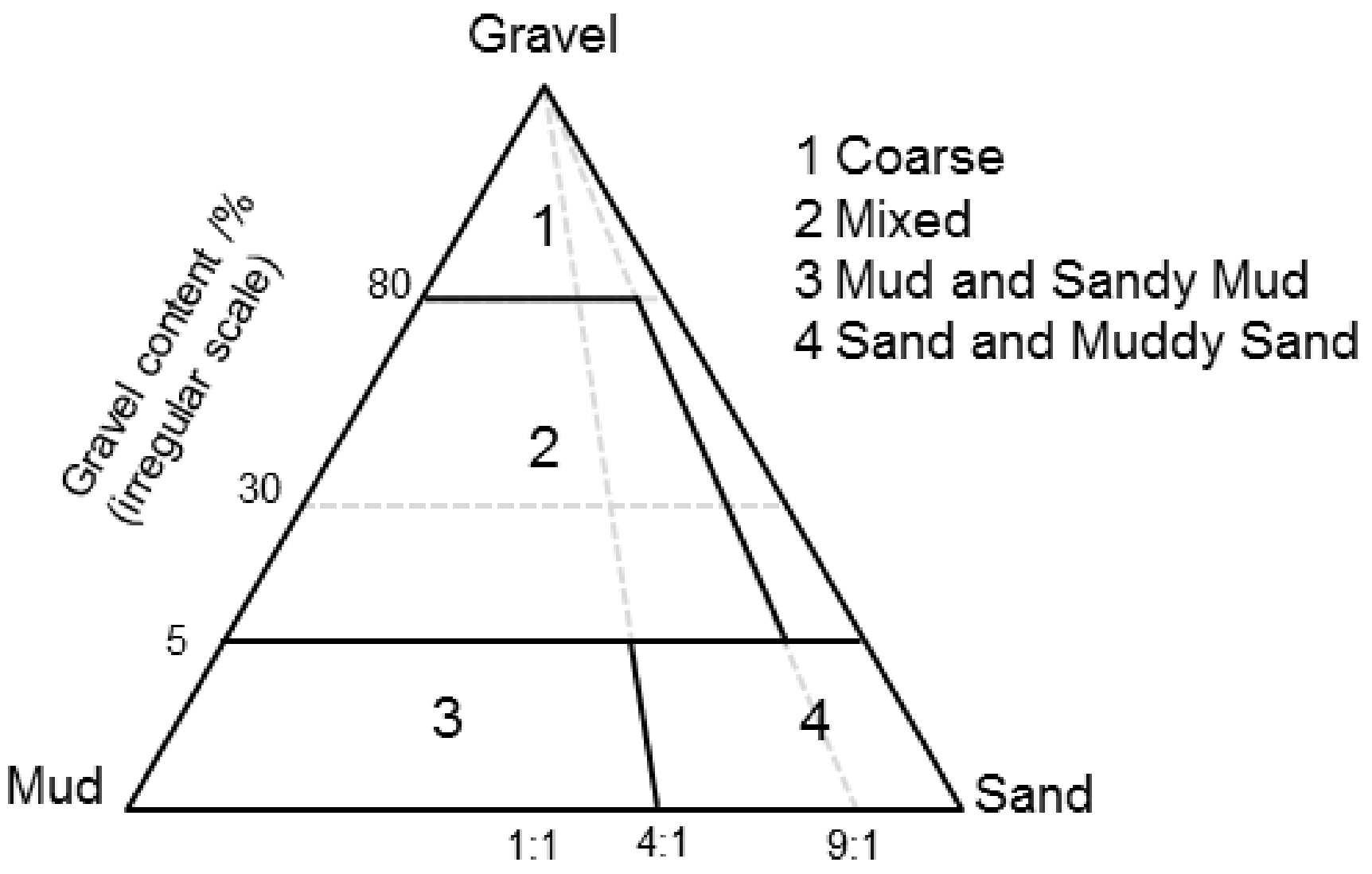

Sand: Mud ratio

(irregular scale) 


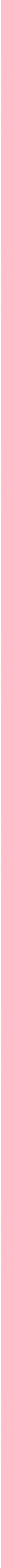




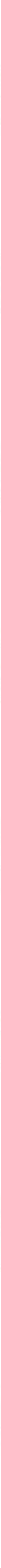



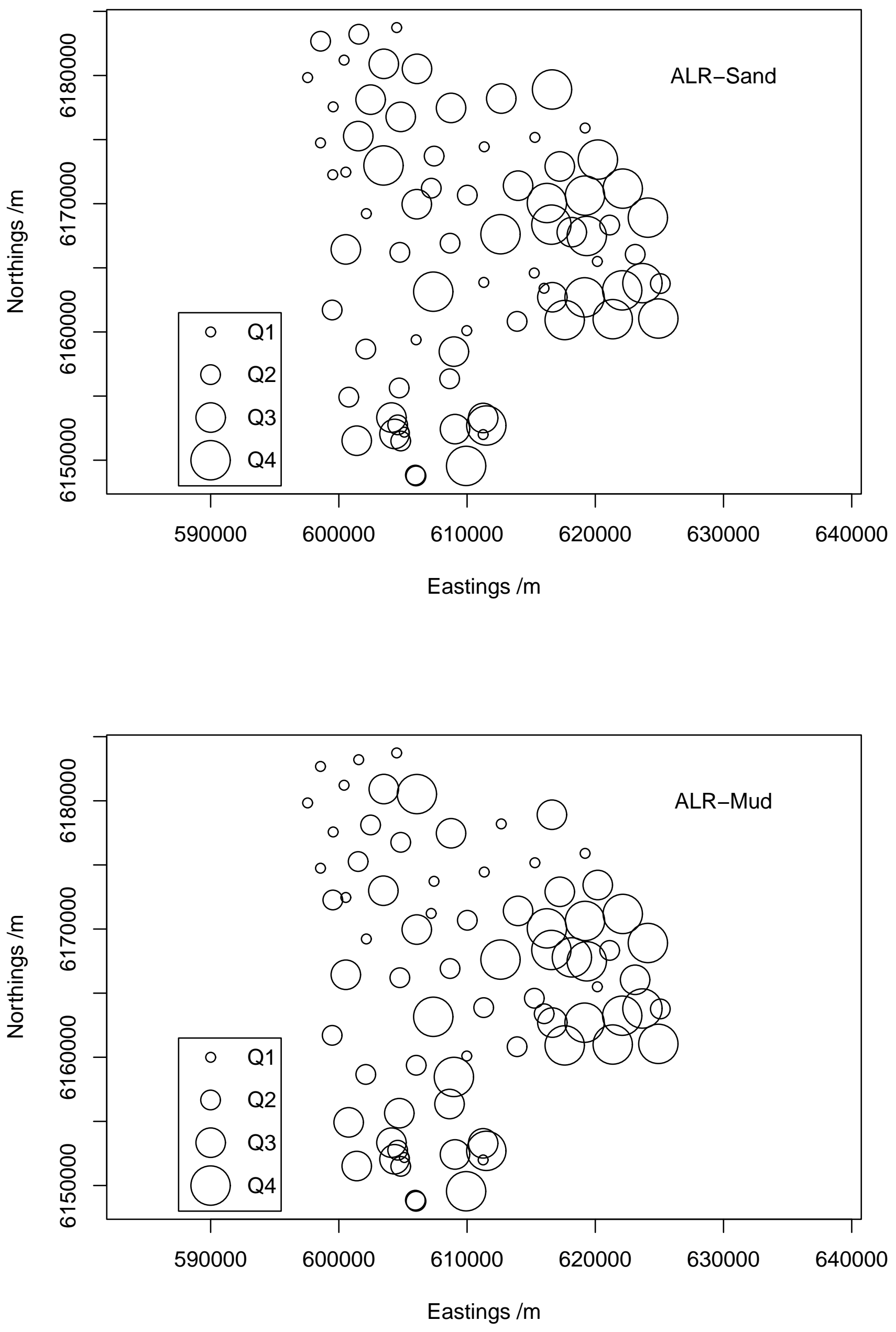

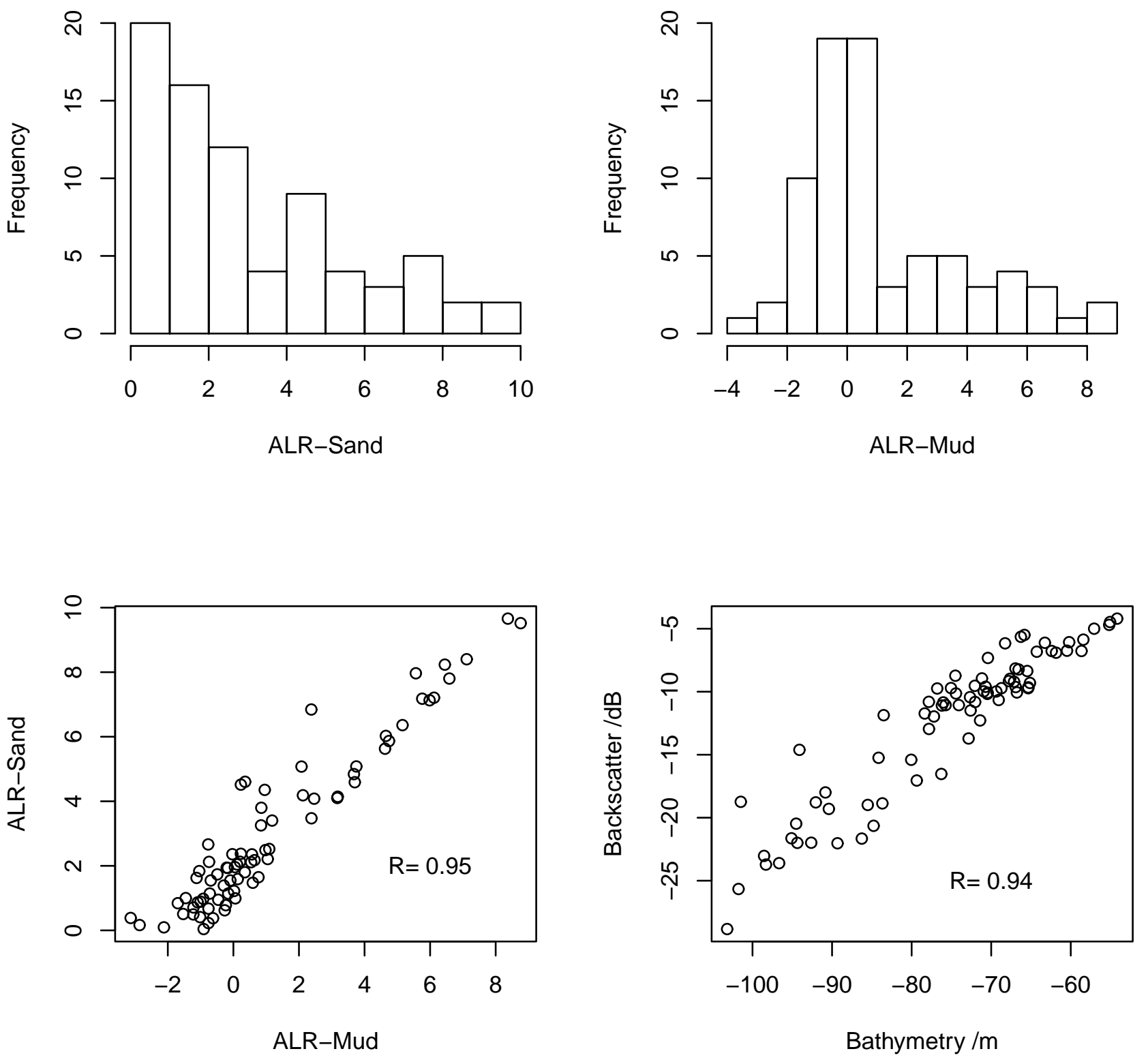

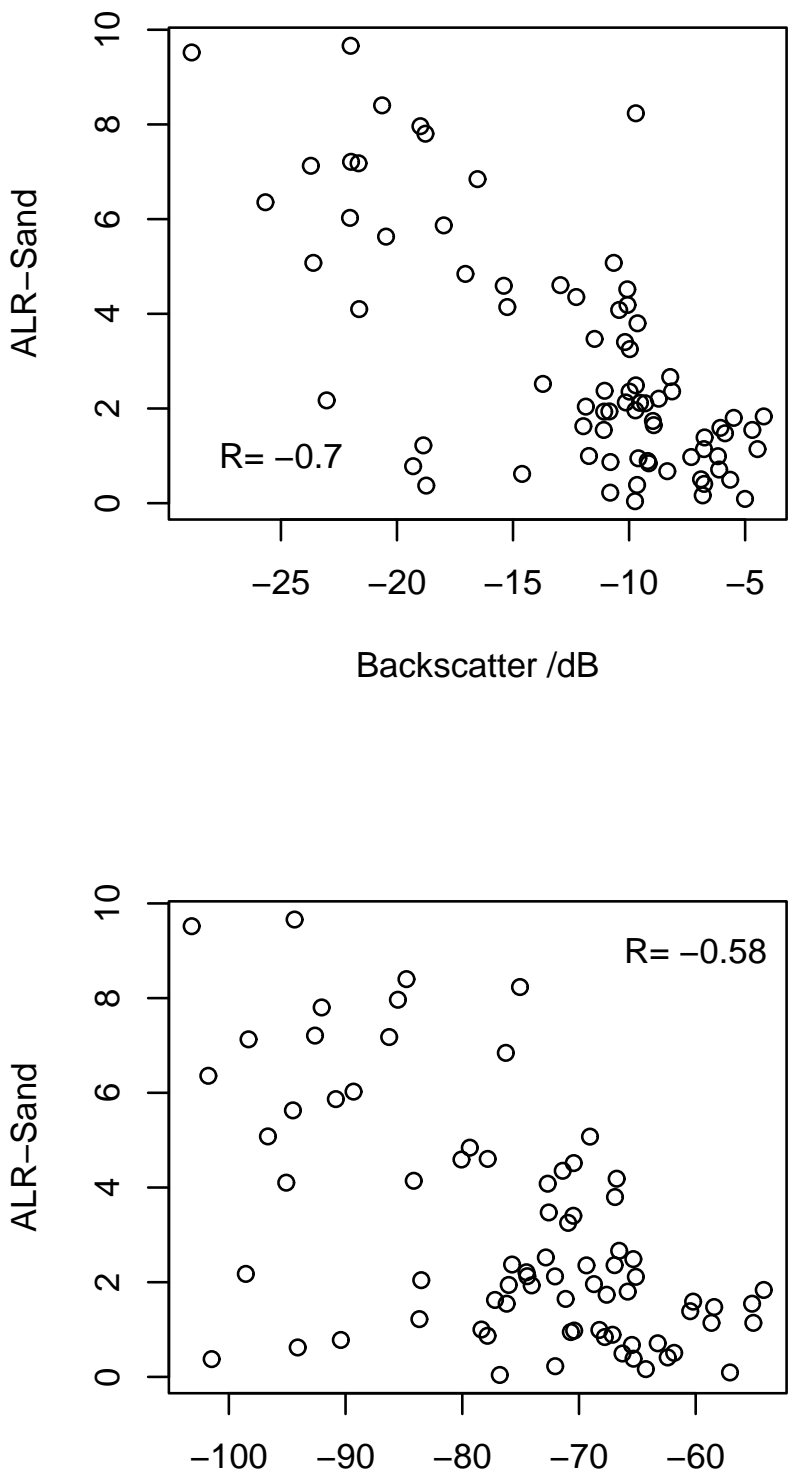

Bathymetry /m
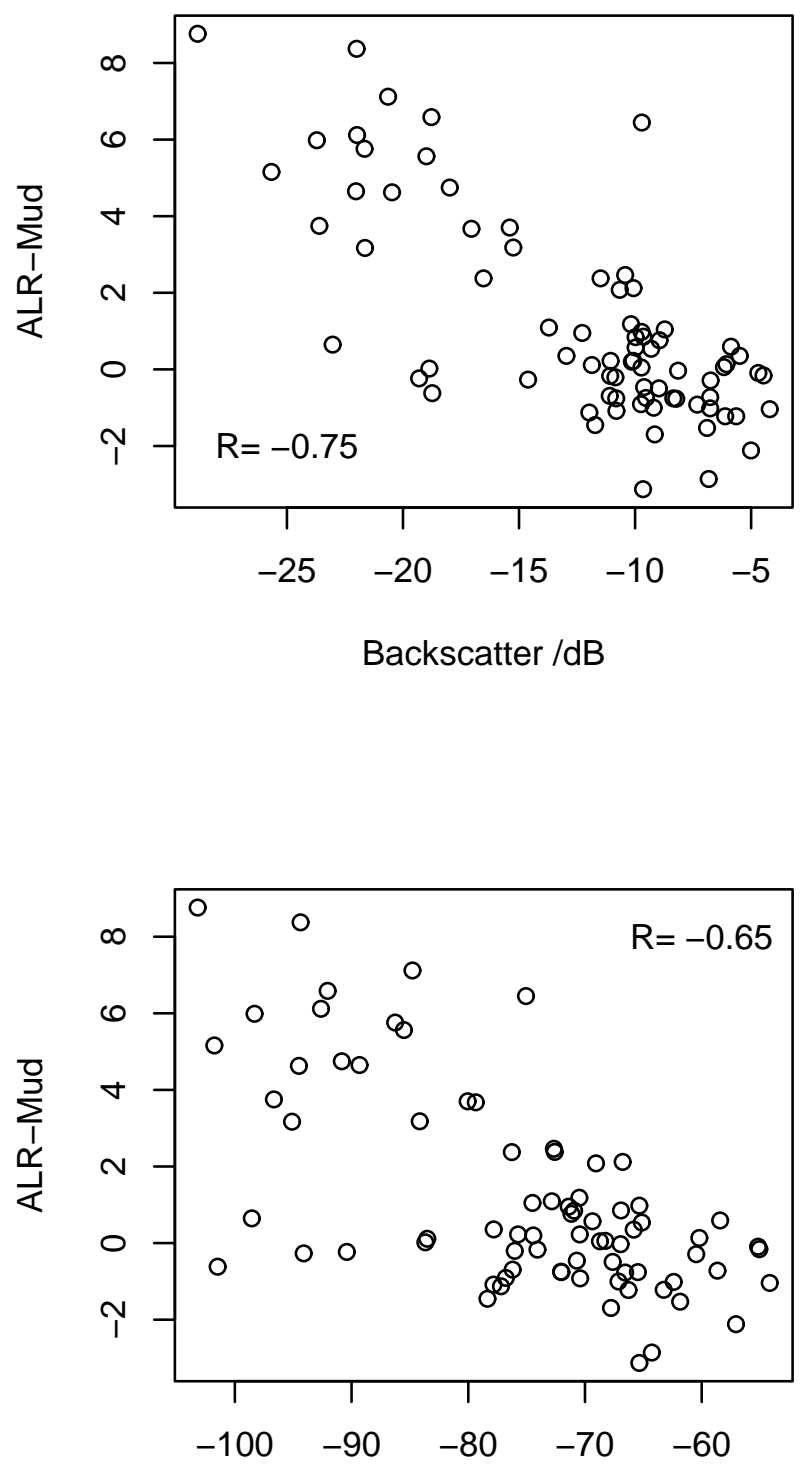

Bathymetry /m 


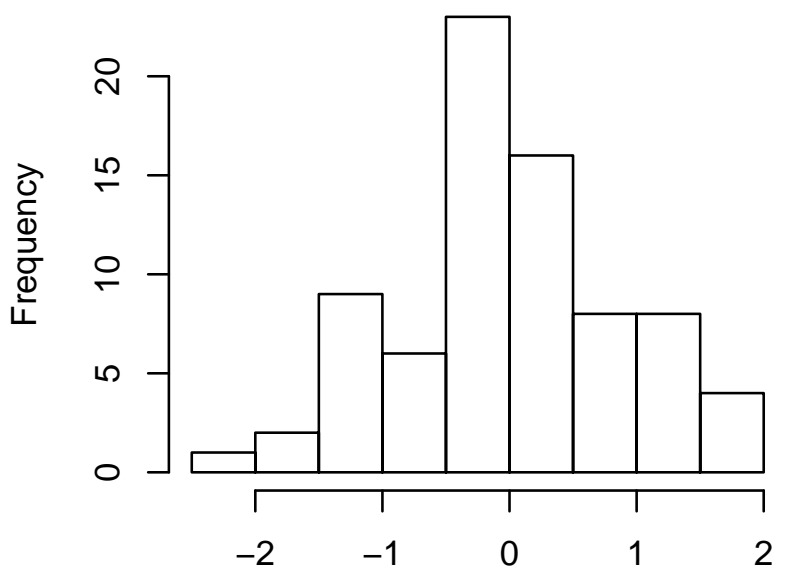

Prediction error, ALR-Sand

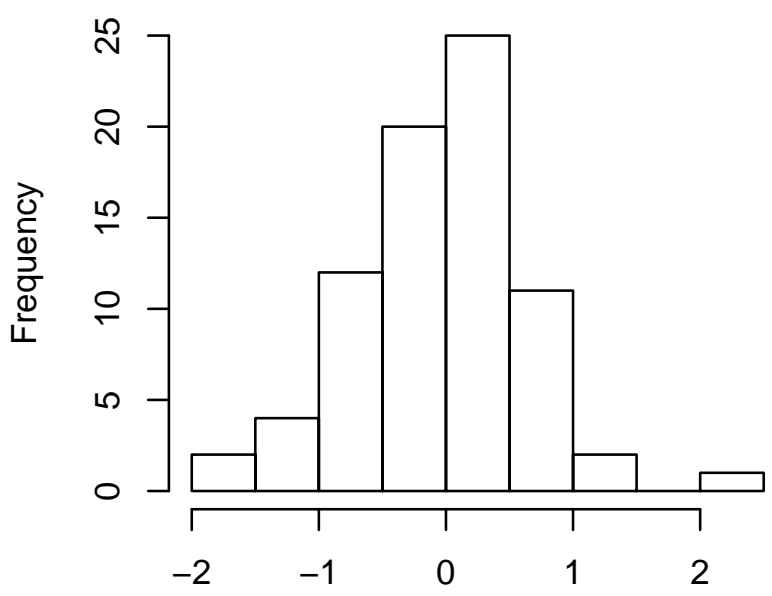

Prediction error, ALR-Sand
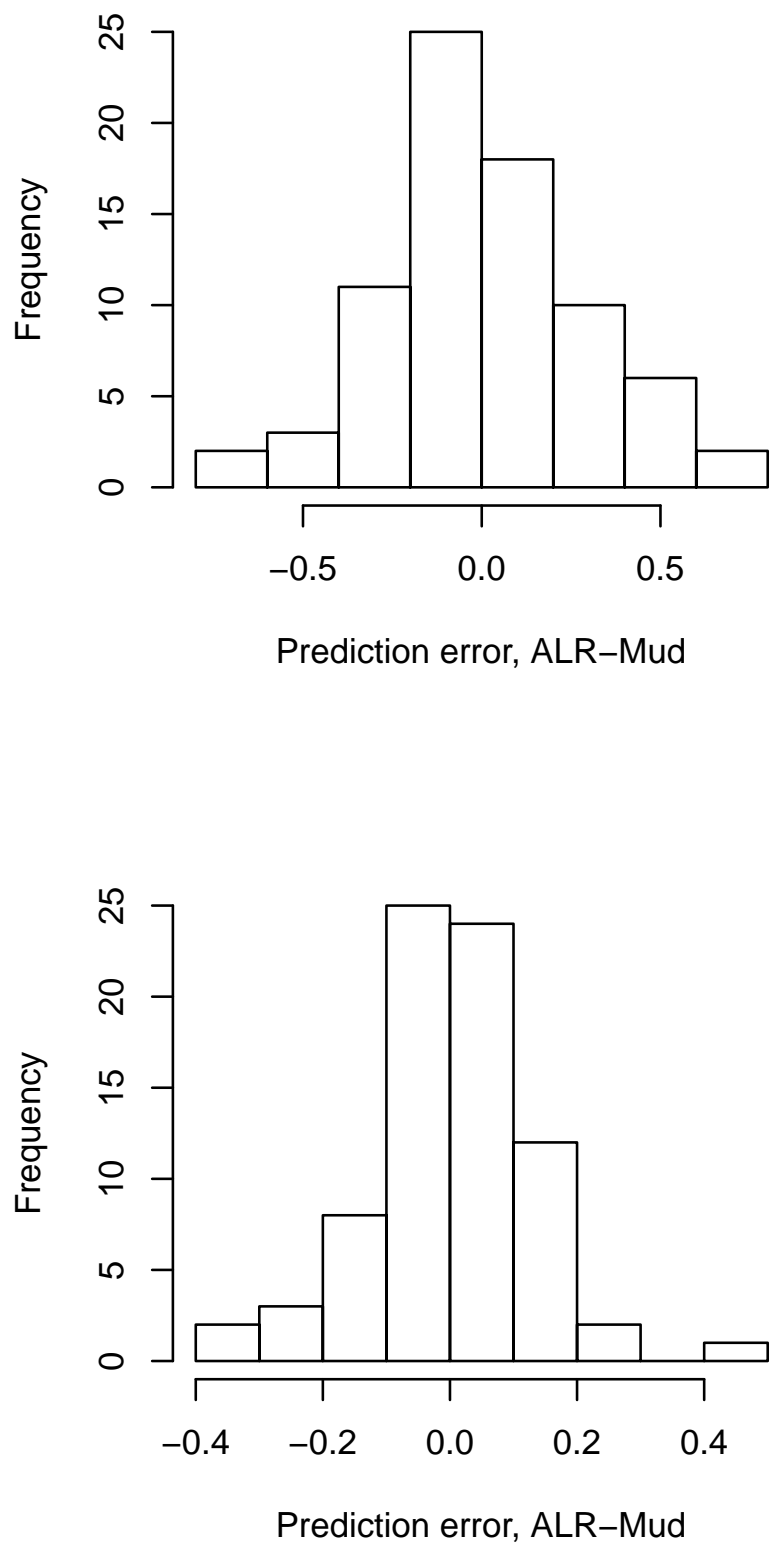

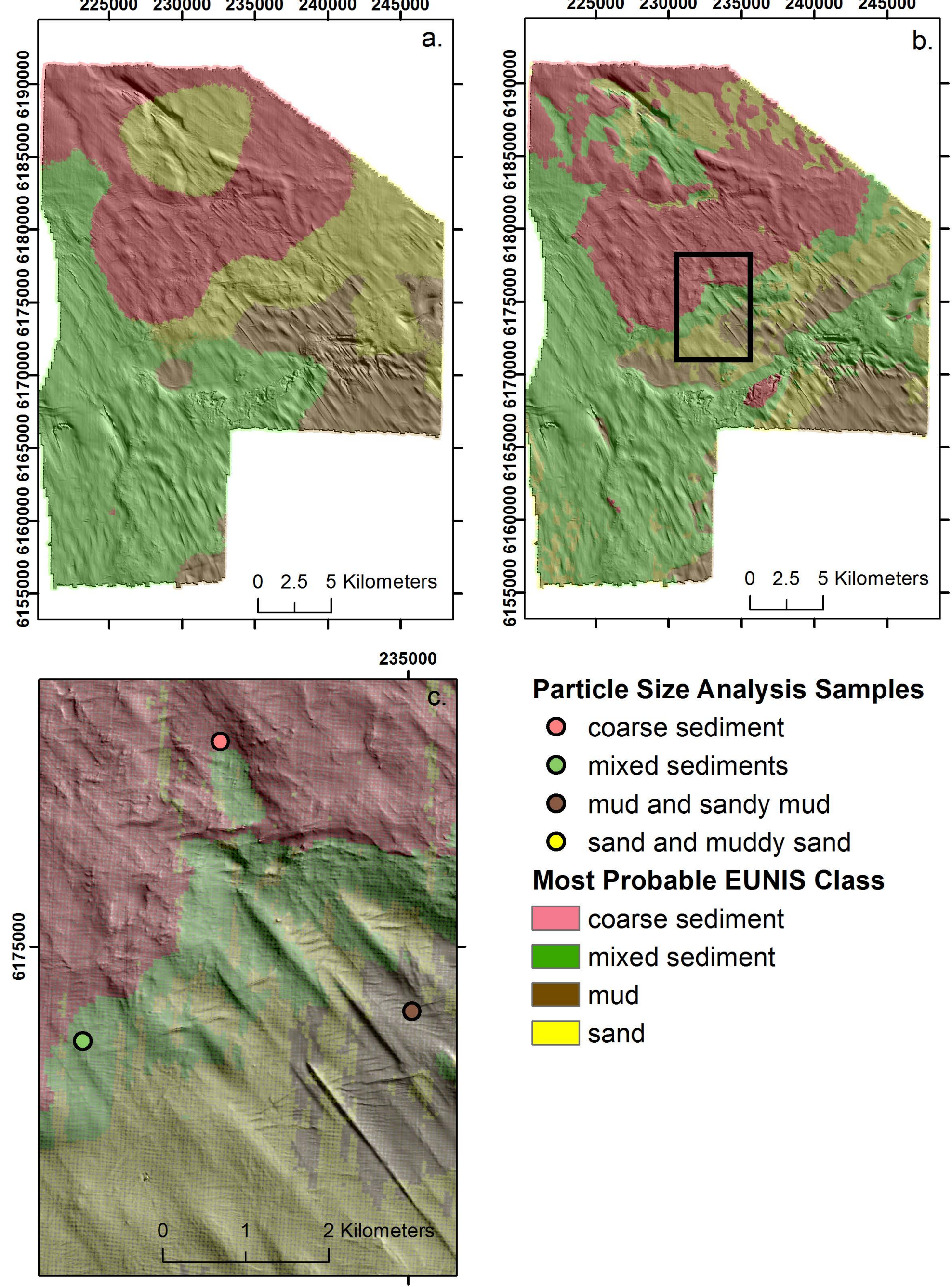

\section{Particle Size Analysis Samples}

O coarse sediment

O mixed sediments

O mud and sandy mud

O sand and muddy sand

\section{Most Probable EUNIS Class}

coarse sediment mixed sediment

mud sand 


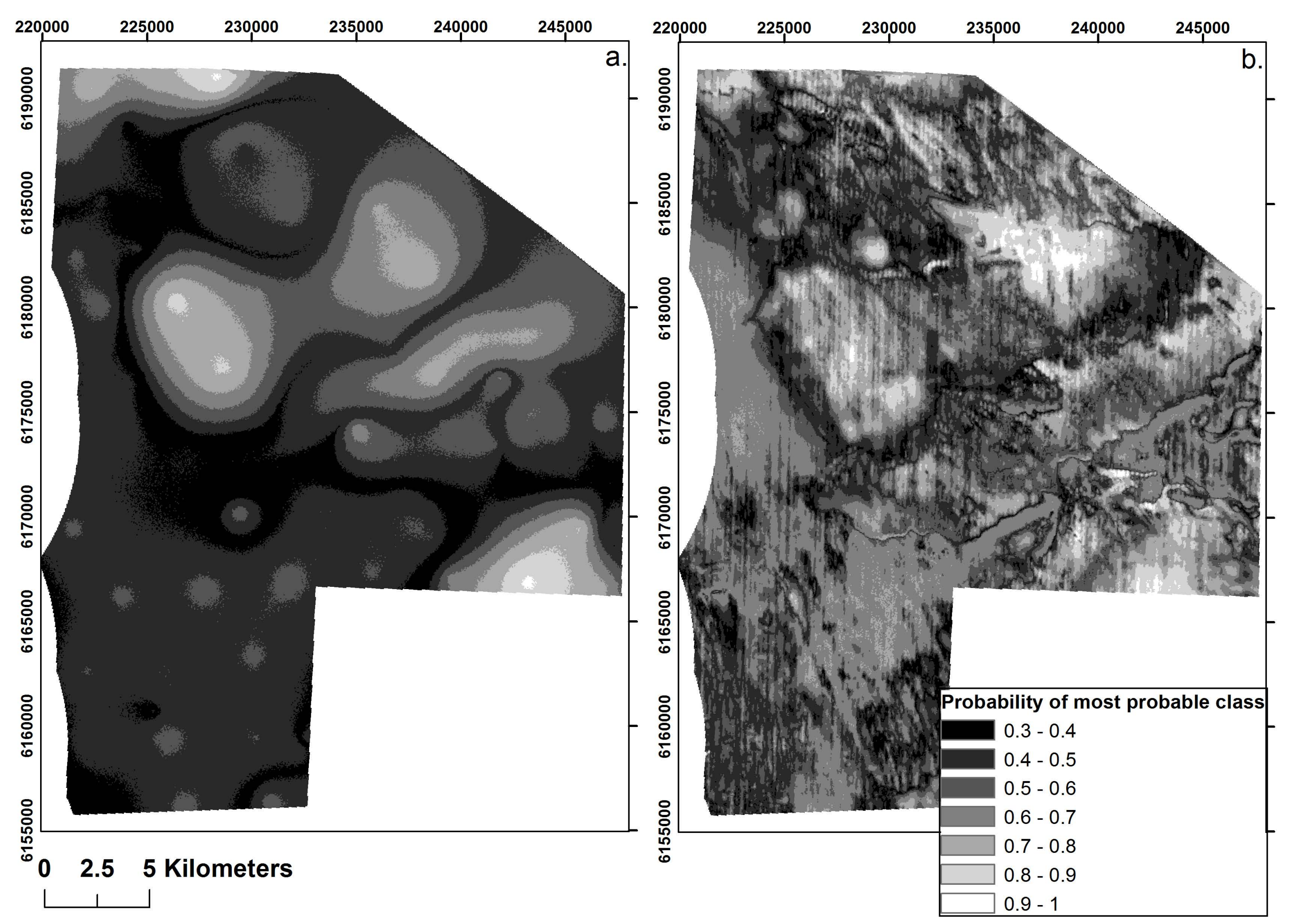




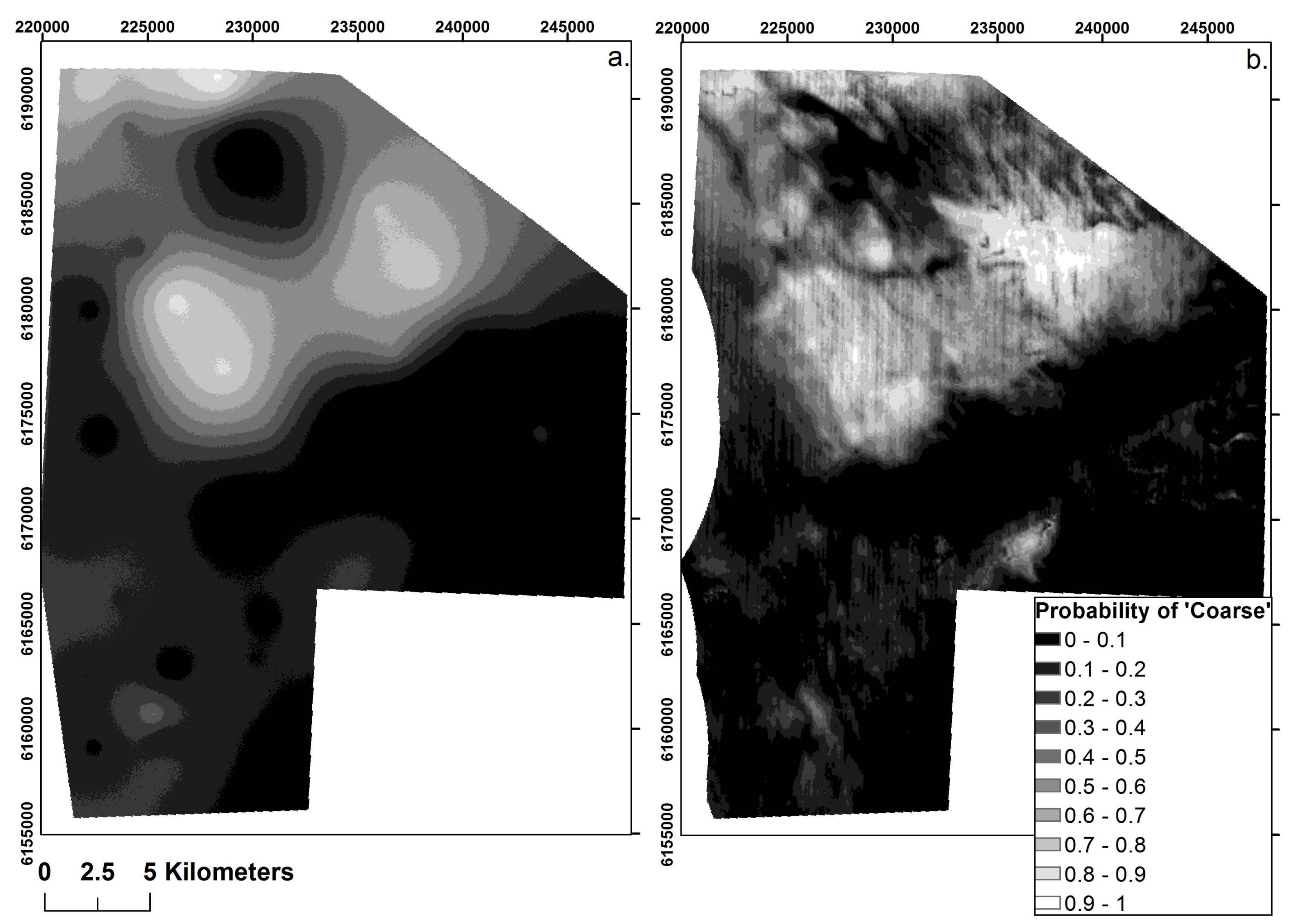


